\title{
Food Additive P-80 Impacts Mouse Gut Microbiota Promoting Intestinal Inflammation, Obesity and Liver Dysfunction
}

\author{
Ratnesh Kumar Singh ${ }^{1 *}$, Seiichi Ishikawa ${ }^{2}$ \\ ${ }^{1}$ Janelia Research Campus, Howard Hughes Medical Institute \\ ${ }^{2} J a p a n$ Science and Technology Agency Kawaguchi, Saitama, Japan
}

Received: April 04, 2016; Accepted: May 27, 2016; Published: June 01, 2016

*Corresponding author: Ratnesh Kumar Singh, Janelia Research Campus, Howard Hughes Medical Institute, Helix Dive, Ashburn, VA 20147 United States, Tel: +1-571-281-0029; Fax: +1-571-551-0107; E-mail: ratneshs@hotmail.com

\begin{abstract}
The increasing prevalence of obesity has emerged as one of the most important global public health issue. The change to the human microbiome as a result of changes in the quality and quantity of food intake over the past several decades has been implicated in the development of obesity and metabolic syndrome. We administered polysorbate-80 to mice via gavage. The researchers monitor liver noninvasively using a bioluminescence imaging. For the liver dysfunction we measure the liver enzymes and PAS stain on liver, electron microscopy liver mitochondria. For the assessment of intestinal inflammation we measured fecal LCN2, LPS, MPO and flagellin by ELISA and qPCR. We use confocal microscopy to detect closet bacteria near the epithelium. 16S sequence was used for the composition of microbiota. Compared with control mice, those receiving emulsifier, showed impaired glycemic tolerance, hyperinsulinemia, altered liver enzymes, larger mitochondria and increased gall bladder size. Additionally, mice in the experimental group showed higher levels of DCA, reduced Muc2 RNA expression, reduced mucus thickness in the intestinal epithelium and increased gut permeability. Intestinal bacteria of mice receiving P-80 were found deeper in the mucus and closer to the intestinal epithelium and had increased level of bioactive LPS, flagellin and LCN2 expression. The result of the study are supportive of evidence that emulsifier agents such as polysorbate-80, may be contributing to obesity related intestinal inflammation and progression of liver dysfunction and alternation of gut microbiota.
\end{abstract}

\section{Abbreviations}

NAFLD: Non Alcoholic Fatty Liver Disease; P-80: Polysorbate 80; LCN2: Lipocalin 2; MUC2: Mucin 2; MPO- Myeloperoxidase; $\mathrm{C}_{\mathrm{T}}$ - Threshold Cycle

\section{Introduction}

The incidence of lifestyle related human disorders has been steadily increasing over the past several decades [1,2]. The rapidity of these developments has been primarily been associated with non-genetic factor such as urbanization and the globalization of unhealthy lifestyle. Key metabolic and physiological changes, including obesity, metabolic syndrome and Nonalcoholic Fatty Liver Disease (NAFLD) are commonly associated with chronic, non-communicable disease that are responsible for approximately $70 \%$ of the world's death.

There are compelling arguments to include the composition of gut microbiota as a key risk factor for the development of obesity and obesity related metabolic disorders, including insulin resistance, type 2 diabetes, dyslipidemia and non-alcoholic fatty liver disease [3]. The gut microbiota has been implicated in having both direct (i.e. through effect on nutrient uptake) and indirect effect on health $[4,5]$. In mice, food additives have been demonstrated to affect the host microbiota interaction, leading to low-grade inflammation, adiposity and adiposity associated metabolic effect [6].

We set out to elucidate whether alteration in the gut microbial community after P-80 ingestion is a causative agent of liver dysfunction. To explore this possibility, we setup an experiment to examine the impact of P-80 on gut microbiota using wild-type C57BL/ 6 mice. The experimental mice were gavaged with the amount of P-80 equal to $1 \%$ of their body weight. This amount correlates to the acceptable daily human intake of $1 \%$ per $\mathrm{kg}$ of polysorbate-80 to body weight [7].

\section{Methods}

\section{Mice and diet}

The C57BL/6(000664) mice were purchased from The Jackson Laboratory. The mice were maintained under specific pathogenfree conditions, on a 12-h light-dark cycle, and fed normal diet (12 kcal\% fat, $29 \mathrm{kcal} \%$ protein, 59\% kcal carbohydrate) ad libitum. For this study there were two groups: one control group (ingesting saline via gavage) and one experimental P-80 fed group (ingesting P-80 dissolve in saline, 1\% per kg via gavage). This dose corresponds to the FDA Acceptable Daily Intake (ADI) in humans (1\% per kg (body weight), adjusted to the mouse 
weight. All animal experiments were cared for under protocols approved by the Committee for the Use and Care of Experimental Animals at the Howard Hughes Medical Institute.

\section{Bioluminescence imaging}

Bioluminescence imaging was performed as previously described in Ooga, et al. [8]. In brief, mice were anesthetized, injected intraperitoneally with D-luciferin sodium salt 5 minutes before beginning photon recording. A luminescence image of the mice was recorded using a cooled, charged-coupled device camera (Princeton instruments). The signal-to-noise ratio was increased by $2 \times 2$ binning and 5 minute exposure.

\section{Analytical procedures}

Blood glucose levels were determined from whole venous blood using an automatic glucose monitor (One Touch). Insulin levels in serum were measured by ELISA using mouse insulin as a standard (Sigma). Triglyceride levels in serum from fasted animals were measured by calorimetric enzyme assay using the GPO-Trinder assay (Sigma). Free fatty acid levels were measured in serum from fasted animals using the NEFA-Kit (Sigma), and liver function tests were measured using a Beckman CX7 analyzer. For the Insulin Tolerance Test (ITT), mice were fasted overnight before intraperitoneal administration of 0.5 or 0.25 unit/ $\mathrm{kg}$ of body weight of insulin. For the pyruvate challenge test, mice were fasted overnight then administrated $2 \mathrm{~g} / \mathrm{kg}$ of body weight of sodium pyruvate by intraperitoneal injection. Blood glucose concentration was measured before and after the injection at the incremental time points. For the measurement of Deoxycholic Acid (DCA), the metabolomics analysis of mice serum was performed by Liquid Chromatography Mass Spectrometry (LCMS) as previously described by Payne, et al. [9].

\section{Histology and electron microscopy}

Tissues were fixed in 10\% buffered formalin and imbedded in paraffin. Staining of liver and intestinal sections with Periodic Acid-Schiff Reagent (PAS) was performed using standard techniques. For electron microscopy, liver was placed in $2 \%$ glutaraldehyde in $0.1 \mathrm{M}$ phosphate buffer ( $\mathrm{pH} 7.4$ ), overnight at $4{ }^{\circ} \mathrm{C}$, then washed and fixed in $2 \% \mathrm{OsO}_{4}$. Section (300-400 A) was stained with saturated uranyl acetate and lead citrate before being examined with a Phillips 301 Transmission Electron Microscope.

\section{Colonic Myeloperoxidase (MPO) Assay}

Neutrophil influx in tissue was analyzed by assaying the enzymatic activity of MPO, a marker for neutrophils according to Chassaing, et al. [10].

\section{In-vivo permeability}

Intestinal barrier function was performed in control and P-80 fed mice using FITC- labeled dextran as previously described by Denizot, et al. [11]. Mice were fasted overnight and then gavaged with $15 \mathrm{mg}$ of FITC-labeled dextran $4 \mathrm{kD}$ (Sigma). Blood was collected after $3 \mathrm{~h}$ and fluorescence intensity was measured in the serum. For tissue studies, Fluorescein isothiocyanate (FITC) dextran $4 \mathrm{kD}$ (Sigma) was added to the to the distal ileum and proximal colon at a concentration of $1 \mathrm{mg} / \mathrm{ml}$. Tissue segments were mounted in Using Chambers with the mucosal medium at each hour for $3 \mathrm{~h}$ after the basolateral medium was sampled. All samples were analyzed for FITC levels with a spectrophotometer microplate reader (excitation 490nm; emission $520 \mathrm{~nm}$ ). FITCdextran concentration was determined using a standard curve calibrated with appropriate standards.

\section{Immunostaining of mucin and localization of bacteria}

Mucus immunostaining was performed as previously described by Johansson, et al. [12]. In brief, the small intestine was isolated; the distal third was dissected and rinsed with PBS. Tissue was then opened longitudinally; rolled mucosa side outwards and colonic tissues containing fecal material were placed in methanol-Carnoy's fixative solution for $3 \mathrm{~h}$ at room temperature. The hybridization step was performed at $50^{\circ} \mathrm{C}$ overnight with the probe 5'-GCTGCCTCCCGTAGGAGT-3' with a 5' Alexa label at a final concentration of $10 \mu \mathrm{g} \mathrm{ml}^{-1}$ in hybridization buffer. Mucin-2 primary antibody (Santa Cruz Biotechnology) was diluted to 1:1000 in block solution and applied overnight at $4^{\circ} \mathrm{C}$. After washing, slides were mounted using Prolong anti-fade mounting media (Life technology). Observations were performed with a Zeiss LSM 700 confocal microscope.

\section{SCFA measurement}

Stool samples were freshly collected and immediately frozen in liquid nitrogen. A 1:5 dilution of the sample in doubled distilled water was centrifuged and the supernatant was mixed with $12 \mathrm{mM}$ isobutyric acid, $1 \mathrm{M} \mathrm{NaOH}$ and $0.36 \mathrm{M} \mathrm{HClO}_{4}$. After lyophilization for $16 \mathrm{~h}$, the remaining powder was diluted with acetone and $5 \mathrm{M}$ formic acid and centrifuged and the supernatant was used for the measurement with an HP 5890 gas chromatography.

\section{Quantitative PCR}

Total RNA was extracted from mouse colonic tissues using TRIZOL reagents (Life Technologies) and reverse transcription and quantitative PCR were performed. Primers used were as follows: Muc2- 5'-CAGCACCGATTGCTGAGTTG-3', and 5'-GCTGGTCATCTCAATGGCAG-3' Lcn-2, 5'-AAGGCAGCTTTACGATGTACAGC-3'and 5'-CTTGCACATTGTAGCTGTGTACC-3'.

\section{S rRNA gene sequence analysis}

Bacterial genomic DNA was isolated from feces using a QIAamp Stool Mini Kit. For amplification of the V1-V4 regions of the 16S rRNA gene, 100 ng of DNA was used on a Genome Sequencer FLX Titanium platform. Sequencing reads were quality filtered, used and subject to Operational Taxonomic Unit (OTU) analysis clustered identified using QIIME software [13] and RDP classifier [14]. The representative sequence from each OTU were blasted to the database of the Ribosomal Database Project (RDP) and aligned. OTU sequence results were grouped and subject to phylogenic analysis using MEGA software as described in Atarashi, et al. [15].

\section{Determination of the copy number of faecal bacteria}

Total bacterial DNA was isolated from weighted faeces 
using DNA Stool Mini Kit (Qiagen) according to manufacturer instructions. Duplicate samples of genomic DNA from each bacterium were amplified by real time PCR (Bio-Rad). The oligonucleotides from Clostridium cluster XIV were used to measure total bacteria [16]. Results were expressed as number of bacteria per mg of stool, using a standard curve as described by Yokoo, et al. [17]. Purified DNA from stool samples were used with optimized PCR conditions and an appropriate standard curve to enumerate the load of different members of Clostridium clusters in the stool samples. Each stool sample was subjected to four PCR runs. The amount of DNA measured by real-time PCR was converted to cell numbers to allow comparison with the CFU data. This was accomplished by using the standard curve that was generated by plotting the CT against CFU. This approach was used because, for stool samples, it is easier to understand results in actual CFU numbers than in DNA concentrations or copy numbers. The CT standard deviations were calculated as shown in Yuli, et al. [18] The CFU of the Clostridium clusters was determined from CT values by using the standard curves.

\section{Statistical analysis}

Significance was analyzed using the t-test, a one-way ANOVA corrected for multiple comparisons with the Sidak test and a two-way ANOVA corrected for multiple comparisons with a Bonferroni test (Graph Pad Prism software). Differences were noted as significant $\leq 0.05$. A nearest-shrunken centroid classification was performed to detect the OTU [19].

\section{Results}

Microbial dysbiosis and inflammation, promoting metabolic syndrome and NAFLD in mice [20] was monitored noninvasively using the bioluminescence imaging technique. The increase in the bioluminescent signal originating from the fatty liver and ballooned hepatocytes (Figure 1) in P-80 fed mice.

The intraperitoneal pyruvate tolerance test was performed on day one with the P-80 fed mice and saline mice showing no differences (data not shown). However, after four weeks of daily P-80 administration, P-80 fed mice demonstrated mild, fasting hyperglycemia and pyruvate intolerance throughout a twohour time period following pyruvate injection (Figure 2A). The intraperitoneal insulin tolerance test (Figure 2B) showed that the P-80 fed mice was significantly more resistant to the blood glucose lowering effect of exogenously administered insulin. Evaluating of the insulin content using acid ethanol extracts demonstrated a twofold higher level of pancreatic insulin in P-80 fed mice have compared to control group of littermates (Figure $2 \mathrm{E})$. To estimate of insulin clearance using a ratio of the serum levels of C-peptide and insulin showed a $60 \%$ insulin clearance reduction compared to the control group of littermates (Figure 2D).

Liver function in P-80 fed mice was assessed by measuring serum levels of albumin and liver enzymes. Serum albumin level was reduced by $50 \%$ in P-80 fed mice (Figure 2C). Liver function tests also revealed a $40 \%$ increase in Alkaline Phosphatase (ALP), a 50\% increase in Aspartate Aminotransferase (AST) and 50\% elevation in Alanine Aminotransferase (ALT) (Figure 2F). Elevated ALP levels are suggestive of biliary tract dysfunction whereas the increased AST and ALT suggest hepatocellular damage. These functional changes in P-80 fed mice were associated with progressive alteration in hepatic morphology. After four weeks of P-80 administration, P-80 fed mice showed steatosis and lipid droplets in histological analysis (Figure $2 \mathrm{~K}$ ). The most striking ultrastructure in P-80 fed mice hepatocytes were the presence of large mitochondria (Figure 2I) compared to control mice. Large

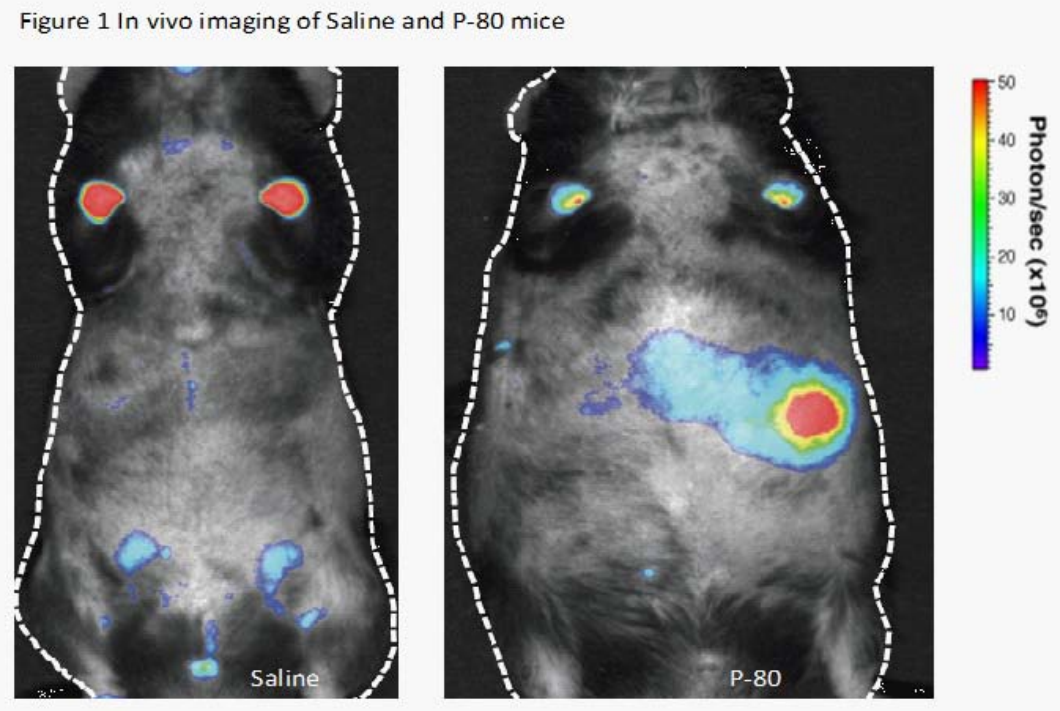

Figure 1: In vivo imaging of saline and P-80 fed mice.

C57BL/ 6 P-80 fed mice and control littermates after 30 days. Real time non-invasive BLI was performed. Representative image of two independent experiments are shown $(n=4)$. 

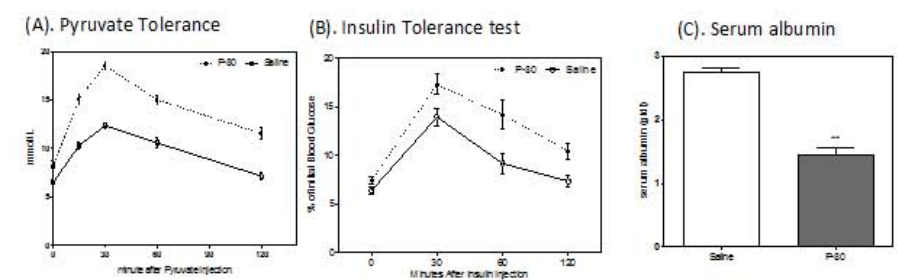

(D). Insulin Clearance

(E.). Insulin Content
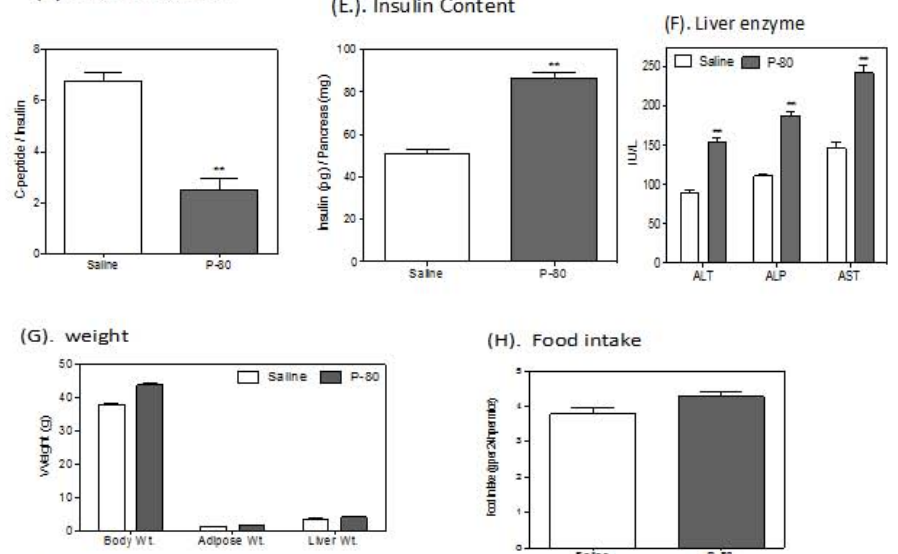

(H). Food intake

(1) Electron microscopic analysis

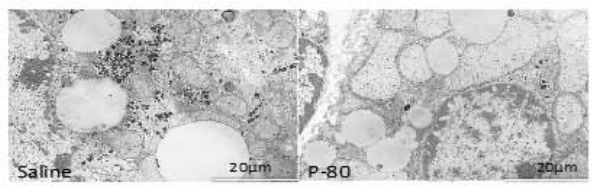

(J)

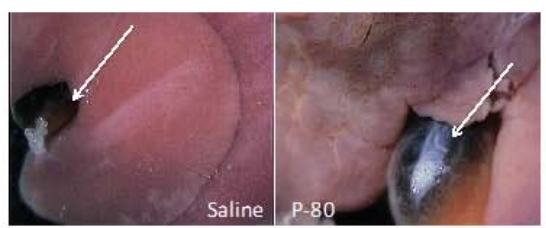

(K) H \& E staining of Adipose and Liver

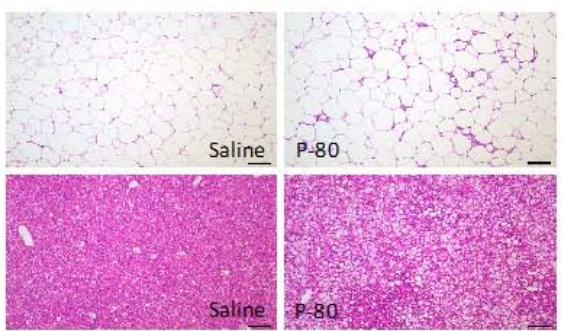

Figure 2: Metabolic parameter in P-80 fed mice.

P-80 promotes metabolic syndrome and liver dysfunction. Animals were injected intraperitoneally $2 \mathrm{~g} / \mathrm{kg}$ body weight of pyruvate. Blood pyruvate was measured immediately before injection and 15, 30, 60 and 120 min after injection. (A) Pyruvate tolerance tests were performed after 4 weeks in P-80 fed mice and control littermates. Insulin tolerance tests were performed. (B) Insulin tolerance test after 4 weeks P-80 fed mice. Serum albumin levels were measured using an albumin determination kit. (C) Serum albumin level after 4 weeks in P-80 fed mice and control littermates. Liver enzyme was measured in the serum of P-80 fed mice. (D) Insulin clearance after 4 weeks P-80 fed mice and control littermates. Insulin content was measured in acid-ethanol extracts. (E) Insulin content after 4 week inP-80 fed mice and control littermates. (F) Liver enzyme after 4 week in P-80 fed mice and control littermates. (G) Body, liver and adipose weight (g) inP-80 fed mice and control littermates. (H) Food intake inP-80 fed mice and control littermates. (I) Electron microscopic analysis of liver section from P-80 fed and control littermates. P-80 fed mice shows giant mitochondria and moderate increase of lipid droplets (J) Gross appearance of P-fed and control mice liver. P-fed mice shows enlarge gallbladder. (K) Representative histology after $\mathrm{H} \& \mathrm{E}$ staining of adipose (upper panel) and liver (lower panel). Data are means \pm s.e. $\mathrm{m}, \mathrm{n}=10$. Significant was determined using oneway ANOVA corrected for multiple comparison with Sidak test. ${ }^{*} P<0.05$ compare to ND treated group. 
mitochondria are observed when there is increased oxidative stress as found with alcoholic liver disease [21]. In addition, the gall bladders of the P-80 fed mice were enlarged (Figure 2J) with an apparent increase in bile volume. P-80 fed mice showed significant gains in weight (Figure 2G) and a marked increased in liver weight and adipose tissue as measured by fat mass. P-80 fed mice also demonstrated increased food consumption (Figure $2 \mathrm{H})$.

\section{Effects of P-80 on colonic mucosa and microbiota localization and pro-inflammatory potential}

Microbiota composition influences the ability of the intestinal microbiome to activate innate immune signaling [22]. P-80 fed mice showed gross histopathological evidence of chronic intestinal inflammation including epithelial damage and a shortened colon (data not shown). The intestinal epithelium is covered by a mucus layer, which is largely composed of mucins and provides a physical barrier, thereby limiting damage to the epithelium and enhancing gut homeostasis by delivering tolerogenic signals [23]. Next we analyzed the pro-inflammatory gene Muc2. In P-80 fed mice, Muc2 mRNA expression is significantly decreased $(40 \%)$ (Figure $3 \mathrm{~A})$. Intestinal permeability was determined by analyzing serum albumin permeability after FITC dextran administration. We found an increase in serum permeability (Figure 3B, C) in P-80 fed mice compared to the control group of littermates. Levels of serum LCN-2 have previously been used as a marker of inflammation [24]. We examined serum LCN-2 and observed a $60 \%$ increase in levels of serum LCN-2 in comparison to control mice (Figure 3D). These results show that serum LCN-2 can detect intestinal inflammation. Colonic expression of LCN-2 mRNA was measured by qRT-PCR and demonstrated a significant increase relative to control mice (Figure 3E). Faecal LCN-2 is a sensitive and broadly dynamic marker of intestinal inflammation in mice [25]. P-80 fed mice showed elevated fecal LCN-2 levels (Figure 3F) after four weeks compared to control mice. We examined the content of feces from P-80 fed mice and control mice for Lipopolysaccharides (LPS) and flagellin. Lipopolysaccharide (LPS) is an endotoxin released by Gramnegative bacteria that can provoke an inflammatory response and thus aggravate inflammation related chronic conditions such as adiposity and insulin resistance. P-80 fed mice showed significant increased levels of bioactive LPS and flagellin compared to control littermates (Figure 3G, H, I). The hallmark of active colitis is the presence of immune cell infiltrates, which is paralleled by change in colon morphology and levels of leukocyte enzyme myeloperoxidase [10]. P-80 fed mice showed increase myeloperoxidase enzyme activity compare to control littermates (Figure 3J). We found that P-80 fed mice had significant reduction in acetate, propionate and butyrate concentrations in fecal samples (Figure 3K).

\section{6rRNA and serum metabolite}

Emerging evidence has indicated that alterations of intestinal microbiota are associated with obesity [26] and metabolic syndrome. Imbalance in the composition of the intestinal microbiota (known as dysbiosis) can be caused by immune deficits and dietary influences [27]. We investigated the possibility that intestinal bacteria play a key role in NAFLD development. Meta $16 \mathrm{~S}$ rRNA gene sequencing analysis of the intestinal microbiota revealed that the percentage of Gram-positive bacterial strains indigenous to the human and rodent intestinal tract [28] was increased in P-80 fed mice compared to the control. Several pathogens have specific strategies for penetrating mucus in order to gain access to epithelial cell surface. Helicobacter pylori uses urease to increase the $\mathrm{pH}$ in its immediate microenvironment, which in turn lowers mucus viscosity allowing the organism to propel itself through the mucus layer [29]. In P-80 fed mice, we found increased levels of Porphyromonadaceae family bacteria in their feces, which is associated with exacerbated hepatic steatosis and inflammation. Campylobacter jejuni and Salmonella spp. use their flagella to penetrate intestinal mucus [30], and levels of these bacteria were found in P-80 fed mice. Additionally, we found a decrease of Bacteroides, which is significant as previous studies found that fecal levels of Bacteroides are decreased in obese patients with type-2 diabetes [18]. We conducted an OTU-based bacterial diversity analysis (Figure 4A). Our results showed an increase number of Helicobacter, Campylobacter jejuni, Porphyromonadaceae family and Salmonella spp. bacteria. A qPCR analysis for Clostridium revealed that the population of cluster XI of genus Clostridium (Figure 4B) was increased in $\mathrm{P}-80$ fed mice compared to control littermates. These results led us to propose that the increase of Gram-positive bacteria may promote NAFLD through the enterohepatic circulation of gut bacterial metabolites. To substantiate this idea we analyzed the serum metabolites of P-80 fed mice and control mice by Liquid Chromatography Mass Spectrometry (LC-MS). We found that the level of Deoxycholic Acid (DCA), a secondary bile acid carried out by gut bacteria such as strains belonging to Clostridium cluster XI and XIV [31] was significantly increased (Figure 4E) by the P-80 fed mice compared to control littermates. Deoxycholic acid is known to cause DNA damage through reactive oxygen species production [32] and is associated with development of NAFLD in both mouse models and humans [33].

\section{Discussion}

The first definitive evidence for the role of gut microbiota in the regulation of host energy homeostasis and adiposity came from Gordon and colleagues [34]. Our study shows that common food additive polysorbate 80 could be contributing the development of chronic inflammatory disease, by disrupting composition of intestinal microbiota, leading to developed NAFLD.

In our experiment, we observed elevated parameters characteristic of the metabolic syndrome. P-80 fed mice showed significant gains in overall weight and marked increase in adiposity as measured by fat mass. Some studies show that the gut microbial community regulates the expression of genes that affect fatty acid oxidation and fat deposition in adipocytes [31]. P-80 fed mice also demonstrated impaired glycemic control as assessed by a fasting pyruvate tolerance test and insulin tolerance testing. P-80 fed mice markedly promoted multiple parameters of metabolic syndrome including body mass, fat mass, food intake, fasting glucose levels and liver dysfunction including liver enzymes, lipid droplets and glucose dysregulation. We also found 
(A)

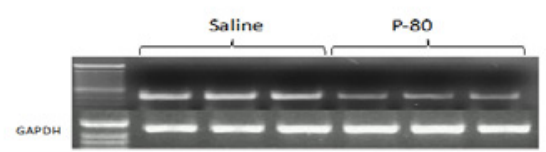

(B)

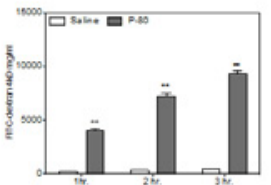

Figure : 3

(c)

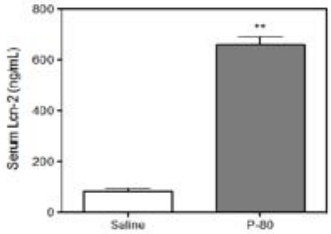

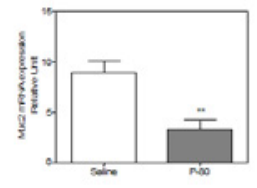

(D)

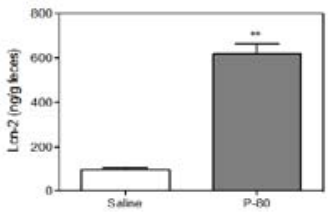

(E)

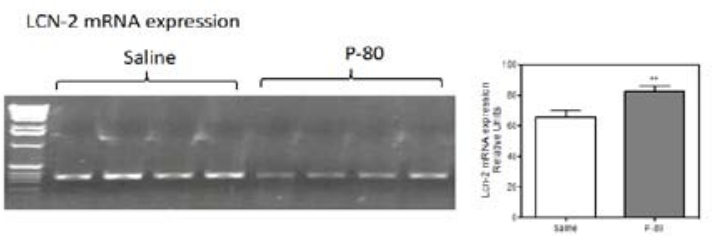

Figure : 3

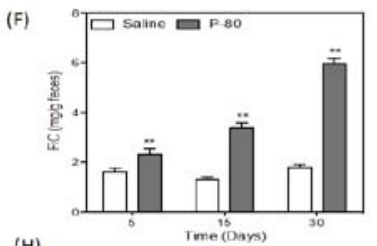

(H)

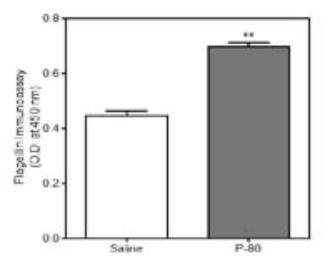

(ग)

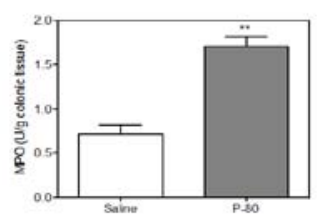

(G)

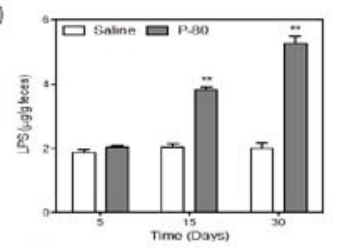

(1)

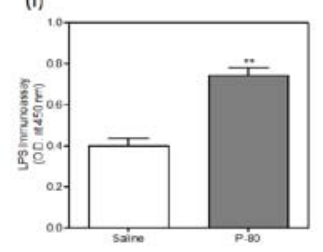

(K)

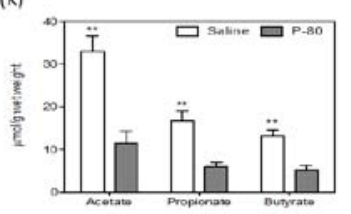

Figure 3: (A) Muc2 mRNA expression in P-80 fed and control littermates. (B) Intestinal permeability measured by levels of serum FITC-dextran (4 $\mathrm{kDa}$ (following oral gavage). (C) Serum LCN-2 level were analyzed by ELISA. P-80 fed mice shows increase Lcn-2 compare to control littermates. (D) Fecal Lcn-2 were measured in feces end of trial by ELISA, P-80 fed mice shows higher expression compare to control littermates. P-80 increases proinflammatory potential of intestinal microbiota compare to control littermates. (E) Colonic Lcn-2 mRNA levels were analysed by qRT-PCR. Bioactive level of faecal flagellin (F) and LPS (G) assayed with TLR5 and TLR4 reporter cells. ( $\mathrm{n}=10)$. P-80 increases serum immune reactivity (IgG) to (H) flagellin and (I) LPS compare to control littermates $(n=10)$. (M) P-80 increase proinflammatory the colonic MPO assay compare to control littermates. (J) An P-80 decreases SCFA concentration: The P-80 decreases the acetate; butyrate and propionate concentration in stool samples in P-80 fed mice control littermates. Data are the means \pm s.e.m. Significance was determined using one-way ANOVA corrected for multiple comparisons. $P<0.05$ compares to control group. 


\section{Figure :4}

(A)

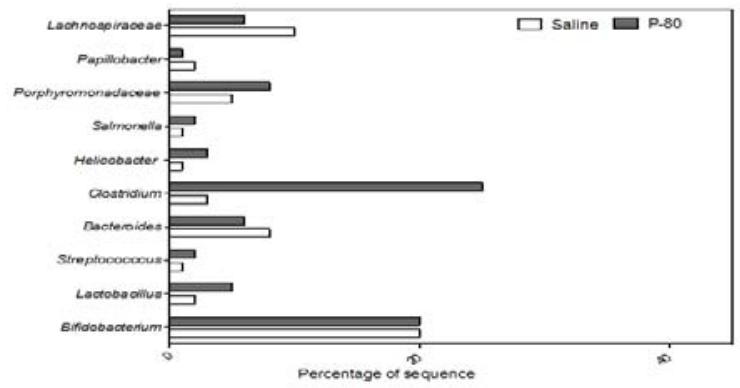

(B)

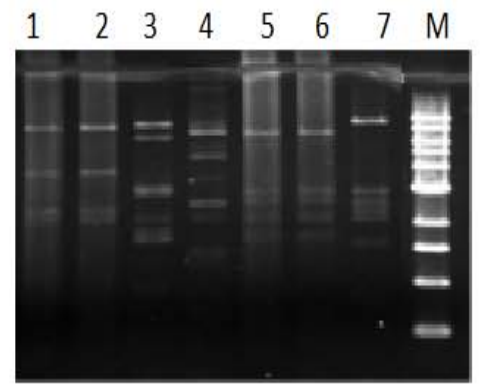

(C)

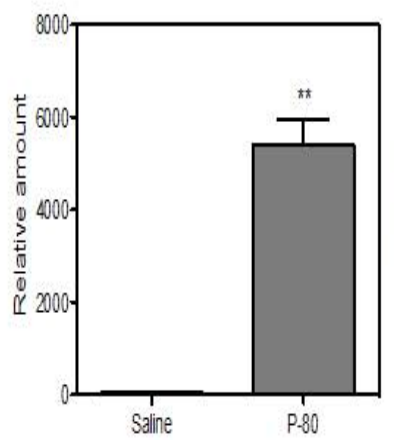

(D)

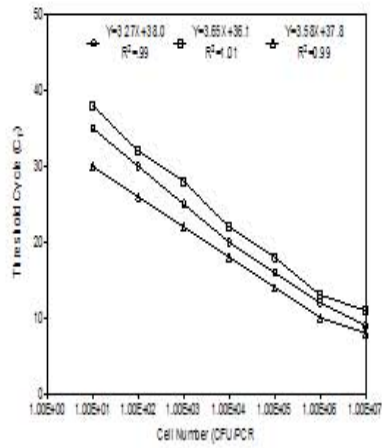

(E)

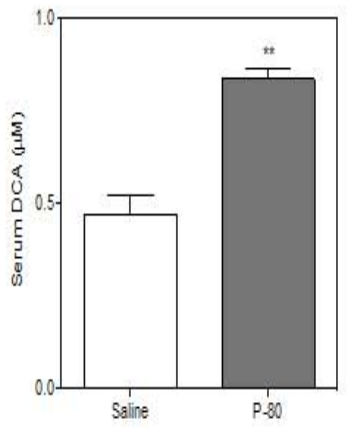

Figure 4: (A) Differentially represented microbiota species analysis from P-80 fed and control littermates and was subjected to amplification of hyper variable region of the bacterial 16S rRNA gene using PCR, followed by pyrosequencing. Sequencing reads were quality filtered, de-multiplexed and assembled to construct OTU by QIIME software with the cutoff similarity of 97\% identity. (B) Agarose gel electrophoresis of PCR product obtained from Clostridium species from P-80 fed mice and control littermates. Lane 1- Clostridium sordellii, lane-2 Clostridium oroticum- DSM 1287, lane-3 Clostridium hylemonae TN-272 JCM 10539, lane-4 Clostridium scindens ATCC 35704, lane-5 Clostridium sp. ID 4 (OTU-95), lane-6 Clostridium innocuum B-3 ATCC 14501, lane-7 Clostridium celerecrescens SCTB 133. (C) Clostridium Cluster is associated with P-80 diet. The qPCR product of clostridium cluster 16 rRNA gene in the feces of control and P-80 fed mice ( $n=5$ per group). (D) Standard curves are generated by analysis of a dilution series of DNA extracted from culture of representative strains for each Clostridium cluster by real time PCR. Quantification was performed by determining the $\mathrm{C}_{\mathrm{T}^{*}}(\mathrm{E})$ Serum DCA concentration (ND, $\mathrm{n}=8, \mathrm{HFD}, \mathrm{n}=8$ ).

enlarged gallbladder size in P-80 fed mice. It is believed that gut bacteria benefit from metabolizing bile acids by acquiring glycine and taurine for subsequent metabolism. However it should be noted that bile acids also exert antimicrobial properties, by being directly toxic to bacteria or by stimulating production of antimicrobial factors [35] and play an important role in preventing small intestinal bacterial overgrowth [36].

P-80 fed mice showed low-grade inflammation as assessed by fecal LCN2 and colon length. This result could reflect direct the effect of P-80 on gut bacteria composition or metabolism. It is noted that SCFA produced by microbial fermentation affect the LPS barrier function [37]. P-80 altered the fecal level of short chain fatty acids and decreased level of butyrate Furusawa, et al. [7] demonstrated that butyrate is known to play a key role in intestinal inflammation [38]. The ingestion of dietary fiber promotes Short Chain Fatty Acid (SCFA) formation and has a beneficial effect on bacteria in gut [39]. Bacteria can serve as an energy source, modulate intestinal motility, are a defense barrier and have been suggested to have an immunoregulatory function 
[40]. The P-80 diet induced NAFLD and intestinal inflammation and was correlated with a significant reduction in acetate, propionate and butyrate concentration in fecal samples. P-80 also altered bile acid levels, which influences microbiota composition [27]. The serum metabolites of P-80 fed mice and control mice were analyzed by LC-MS. Interestingly the level of DCA a secondary bile acid carried out by gut bacteria such as Clostridium Cluster XI and XIV was substantially increased. We found that after P-80 feeding, mice demonstrated an increase the intestinal permeability. It is well recognized that natural detergents in the form of bile acids can increase intestinal permeability [41] and non-ionic detergents have been shown to increase permeability across intestinal epithelial cell monolayers [40]. Confocal microscopy using mucus-preserving Carnoy Fixation [42] indicated that the closest bacteria reside, on average, about $25 \mu \mathrm{m}$ from epithelial cells. P-80 fed mice exhibited some bacteria in contact with the epithelium and the average distance was reduced by $40 \%$. Such microbiota encroachment correlated with reduced mucus thickness. Microbiota composition influences the ability of a microbiota to activate innate immune signaling [22]. Thus, we measured the capacity of feces from control and P-80 fed mice to activate pro-inflammatory cytokines the Lipopolysaccharide (LPS) and flagellin receptors. Exposures to P-80 increased levels of bioactive LPS and flagellin. P-80 also increased gut permeability, which correlated with increased level of serum flagellin and LPS, though to reflect gut permeability [12]. Thus $\mathrm{P}-80$ results in erosion of the protective function of the mucus, increased bacterial adherence and a more pro-inflammatory microbiota.

In regards to microbiota composition a reduced level of Bacteroides is associated with health [43] and increased level of mucolytic operational taxonomic units, including Ruminococcus gnavus [44]. Notably, Operational Taxonomic Unit (OTU) based bacterial diversity analysis in conjunction with a quantitative PCR analysis revealed that the population of Clostridium cluster $\mathrm{XI}$ was strikingly increased in P-80 fed mice. After P-80 ingestion, we found significant difference in the structure of the enteric microbiota as assessed by 16S ribosomal RNA (rRNA) libraries from stool. Gram-negative bacteria are unlikely to promote NAFLD development. Indeed, Meta 16S rRNA gene sequencing analysis of the intestinal microbiota revealed that the percentage of Gram-positive bacterial strains indigenous to the human and rodent intestinal tract was increased with a P-80 diet. These results lead us to propose that the increase of Gram-positive bacteria may promote NAFLD development, presumably through the enterohepatic circulation of gut bacterial metabolites or toxins.

Over the last few decades, consumption of food additives has increased. Most of these additives have not been thoroughly tested given GRAS "ingredients generally recognized as safe" status by food controllers. The majority of testing of the food additives was performed on animal models for the detection and /or promotion of cancer and toxicity [39]. Such testing may be inadequate as suggested by a recent study demonstrated that artificial sweeteners induce dysglycemia in humans [45].
Our data suggest that one such food additive P-80 can disturb the host gut microbiota relationship resulting in a microbiota with enhanced mucolytic and pro-inflammatory activity that promotes intestinal inflammation. Such inflammation and bile acid regulation manifests as dysbiosis, metabolic syndrome and NAFLD. While additional studies will be needed to determine the effect of P-80 emulsifier on human health, our finding in mice suggest that the metabolic syndrome, obesity and NAFLD may be driven, in part by food additive and other factors that might alter gut microbiota and promote intestinal inflammation.

\section{Acknowledgement}

We thank to Dr. Mario R. Capecchi for guidance and data interpretation. Dr. Oliver Baclic and Christina Jensen for editing the manuscript.

\section{Disclosures}

PCR- derived 16S rRNA gene sequences are deposited in GenBank under the accession numbers EE94963-110119. The authors declare no competing financial interests.

\section{Grants}

This work was supported by the National Institute of Health (NIH) through grant DK093243 to R.K. Singh, as well as by a grant from Japan Science and Technology of Japan (JST) to Seiichi Ishikawa.

\section{Author Contributions}

R.K. Singh conceived the project, designed and performed the experiments, analyzed the data and wrote the manuscript. $\mathrm{N}$. Wheildon performed the liver histology experiments. S. Ishikawa performed the bacterial genome data analysis.

\section{References}

1. Wang Q, Garrity GM, Tiedje JM, Cole JR. Naive Bayesian classifier for rapid assignment of rRNA sequence into the new bacterial taxonomy. Appl Environ Microbiol. 2007;73(16):5261-7.

2. NHANES. Trends in intake of energy and macronutrients- United States, 1971-2000. 2004;53(04):80-82. http://www.cdc.gov/mmwr/ preview/mmwrhtml/mm5304a3.htm

3. Turnbaugh PJ, Ley RE, Mahowald MA, Magrini V, Mardis ER, Gordon JI. An obesity-associated gut microbiome with increased capacity for energy harvest. Nature. 2006;444(7122):1027-31.

4. Lorenzo-Zúñiga $V$, Bartolí R, Planas R, Hofmann AF, Viñado B, Hagey $L R$, et al. Oral bile acids reduce bacterial overgrowth, bacterial translocation and endotoxemia in cirrhotic rats. Hepatology. 2003;37(3):551-7.

5. Turnbaugh PJ, Hamady M, Yatsunenko T, Cantarel BL, Duncan A, Ley $\mathrm{RE}$, et al. A core gut microbiome in obese and lean twins. Nature. 2009;457(7228):480-4. doi: 10.1038/nature07540.

6. Caporaso JG, Kuczynski J, Stombaugh J, Bittinger K, Bushman FD, Costello EK, et al. QIIME allows analysis of high-throughput community sequencing data. Nat Methods. 2010;7(5):335-6. doi: 10.1038/nmeth.f.303.

7. Furusawa Y, Obata Y, Fukuda S, Endo TA, Nakato G, Takahashi D, et al. Commensal microbe-derived butyrate induces the differentiation 
of colonic regulatory T cells. Nature. 2013;504(7480):446-50. doi: $10.1038 /$ nature 12721

8. Ooga T, Sato H, Nagashima A, Sasaki K, Tomita M, Soga T, et al Metabolomic anatomy of an animal model revealing homeostatic imbalances in dyslipidaemia. Mol Biosyst. 2011;7(4):1217-23. doi: $10.1039 / \mathrm{c} 0 \mathrm{mb} 00141 \mathrm{~d}$

9. Payne CM, Weber C, Crowley-Skillicorn C, Dvorak K, Bernstein $\mathrm{H}$ Bernstein $\mathrm{C}$, et al. Deoxycholate induces mitochondrial oxidative stress and activates NF-kappaB through multiple mechanisms in HCT116 colon epithelial cells. Carcinogenesis. 2007;28(1):215-22.

10. Chassaing B, Koren O, Goodrich JK, Poole AC, Srinivasan S, Ley RE, et al. Dietary emulsifier impact the mouse gut microbiota promoting colitis and metabolic syndrome. Nature. 2015;519(7541):92-6. doi: 10.1038/nature14232.

11. Denizot J, Sivignon A, Barreau F, Darcha C, Chan HF, Stanners CP, et al. Adherent-invasive Escherichia coli induce claudin-2 expression and barrier defect in CEABAC10 mice and Crohn's disease patients. Inflamm Bowel Dis. 2012;18(2):294-304. doi: 10.1002/ibd.21787.

12. Johansson ME, Hansson GC. Preservation of mucus in histological sections, immunostaining of mucins in fixed tissue, and localization of bacteria with FISH. Methods Mol Biol. 2012;842:229-35. doi: 10.1007/978-1-61779-513-8_13.

13. Chassaing B, Koren O, Carvalho FA, Ley RE, Gewirtz AT. AIEC pathobiont instigates chronic colitis in susceptible hosts by altering microbiota composition. Gut. 2014;63(7):1069-80. doi: 10.1136/ gutjnl-2013-304909.

14. Wu X, Ma C, Han L, Nawaz M, Gao F, Zhang X, et al. Molecular characterisation of the faecal microbiota in patients with type II diabetes. Curr Microbiol. 2010;61(1):69-78. doi: 10.1007/s00284010-9582-9.

15. Atarashi K, Tanoue T, Shima T, Imaoka A, Kuwahara T, Momose $\mathrm{Y}$, et al. Induction of colonic regulatory $\mathrm{T}$ cells by indigenous Clostridium species. Science. 2011;331(6015):337-41. doi: 10.1126/ science.1198469.

16. Tibshirani R, Hastie T, Narasimhan B, Chu G. Diagnosis of multiple cancer types by shrunken centroids of gene expression. Proc Natl Acad Sci U S A. 2002;99(10):6567-72.

17. Yokoo H, Singh SK, Hawasli AH. Giant mitochondria in alcoholic liver disease. Arch Pathol Lab Med. 1978;102(4):213-4.

18. Song Y, Liu C, Finegold SM. Real time PCR quantitation of Clostridia in Feces of Autistic children. Appl Environ Microbiol. 2004;70(11):645965.

19. Vijay-Kumar M, Wu H, Jones R, Grant G, Babbin B, King TP, et al Flagellin suppresses epithelial apoptosis and limits disease during enteric infection. Am J Pathol. 2006;169(5):1686-700.

20. García-Ruiz C, Baulies A, Mari M, García-Rovés PM, Fernandez-Checa JC. Mitochondrial dysfunction in non-alcoholic fatty liver disease and insulin resistance: cause or consequence? Free Radic Res. 2013;47(11):854-68. doi: 10.3109/10715762.2013.830717.

21. Ziegler TR, Luo M, Estívariz CF, Moore DA, Sitaraman SV, Hao L, et al Detectable serum flagellin and lipopolysaccharide and upregulated anti-flagellin and lipopolysaccharide immunoglobulins in human short bowel syndrome. Am J Physiol Regul Integr Comp Physiol. 2008;294(2):R402-10

22. Chassaing B, Srinivasan G, Delgado MA, Young AN, Gewirtz AT, Vijay-Kumar M. Fecal lipocalin 2, a sensitive and broadly dynamic non-invasive biomarker for intestinal inflammation. PLoS One. 2012;7(9):e44328. doi: 10.1371/journal.pone.0044328.

23. Suez J, Korem T, Zeevi D, Zilberman-Schapira G, Thaiss CA, Maza O, et al. Artificial sweeteners induce glucose intolerance by altering the gut microbiota. Nature. 2014;514(7521):181-6. doi: 10.1038/ nature13793.

24. Walter J, Ley R. The human gut microbiome: ecology and recent evolutionary changes. Annu Rev Microbiol. 2011;65:411-29. doi: 10.1146/annurev-micro-090110-102830.

25. Celli JP, Turner BS, Afdhal NH, Keates S, Ghiran I, Kelly CP, et al. Helicobacter pylori moves through mucus by reducing mucin viscoelasticity. Proc Natl Acad Sci U S A. 2009;106(34):14321-6. doi: 10.1073/pnas.0903438106.

26. Turnbaugh PJ, Bäckhed F, Fulton L, Gordon JI. Diet-induced obesity is linked to marked but reversible alternation in the mouse distal gut microbiome. Cell Host Microbe. 2008;3(4):213-23. doi: 10.1016/j. chom.2008.02.015.

27. Dimitrijevic D, Shaw AJ, Florence AT. Effects of some non-ionic surfactants on transepithelial permeability in Caco-2 cells. J Pharm Pharmacol. 2000;52(2):157-62.

28. Shan M, Gentile M, Yeiser JR, Walland AC, Bornstein VU, Chen K, et al. Mucus enhances gut homeostasis and oral tolerance by delivering immunoregulatory signals. Science. 2013;342(6157):447-53. doi: 10.1126/science.1237910.

29. Hofmann AF, Eckmann L. How bile acids confer gut mucosal protection against bacteria. Proc Natl Acad Sci U S A. 2006;103(12):4333-4.

30. Brun P, Castagliuolo I, Di Leo V, Buda A, Pinzani M, Palù G, et al. Increased intestinal permeability in obese mice: new evidence in the pathogenesis of nonalcoholic steatohepatitis. Am J Physiol Gastrointest Liver Physiol. 2007;292(2):G518-25.

31. Png CW, Lindén SK, Gilshenan KS, Zoetendal EG, McSweeney CS, Sly LI, et al. Mucolytic bacteria with increased prevalence in IBD mucosa augment in vitro utilization of mucin by other bacteria. Am J Gastroenterol. 2010;105(11):2420-8. doi: 10.1038/ajg.2010.281.

32. Guerry P. Campylobacter flagella: not just for motility. Trends Microbiol. 2007;15(10):456-61.

33. Bäckhed F, Ding H, Wang T, Hooper LV, Koh GY, Nagy A, et al. The gut microbiota as an environmental factor that regulates gat storage. Proc Natl Acad Sci U S A. 2004;101(44):15718-23.

34. Henao-Mejia J, Elinav E, Jin C, Hao L, Mehal WZ, Strowig T, et al. Inflammasome-mediated dysbiosis regulates progression of NAFLD and obesity. Nature. 2012;482(7384):179-85. doi: 10.1038/ nature10809.

35. Maslowski KM, Mackay CR. Diet, gut microbiota and immune responses. Nat Immunol. 2011;12(1):5-9. doi: 10.1038/ni0111-5.

36. Castaneda FE, Walia B, Vijay-Kumar M, Patel NR, Roser S, Kolachala $\mathrm{VL}$, et al. Targeted deletion of metalloproteinase 9 attenuates experimental colitis in mice: central role of epithelial- derived MMP. Gastroenterology. 2005;129(6):1991-2008.

37. Ridlon JM, Kang DJ, Hylemon PB. Bile salt biotransformations by human intestinal bacteria. J Lipid Res. 2006;47(2):241-59.

38. Food and Agriculture Organisation/World Health Organisatio (FAO/ WHO) expert committee on food additives. Toxicology evaluation of some food additives including anticaking agents, antimicrobial, antioxidants, emulsifiers and thickening agents. WHOFood additives Sedrioes. 1974;5:254-63. 
39. Freel RW, Hatch M, Earnest DL, Goldner AM. Role of tight-junction pathways in bile salt- induced increases in colonic permeability. Am J Physiol. 1983;245(6):G816-23.

40. Gregor MF, Hotamisligil GS. Inflammatory mechanisms in obesity. Annu Rev Immunol. 2011;29:415-45. doi: 10.1146/annurevimmunol-031210-101322.

41. Ley RE, Turnbaugh PJ, Klein S, Gordon JI. Microbial ecology: human gut microbes associated with obesity. Nature. 2006;444(7122):10223.
42. Redgwell RJ, Fischer M. Dietary fiber as a versatile food component: an industrial perspective. Mol Nutr Food Res. 2005;49(6):521-35.

43. Qin J, Li R, Raes J, Arumugam M, Burgdorf KS, Manichanh C, et al. A human gut microbial gene catalogue established by metagenomic sequencing. Nature. 2010;464(7285):59-65. doi: 10.1038/ nature 08821. 